\title{
An image-based eyelid lesion management service- evaluation of a pilot
}

\author{
J. Hind $\mathbb{D}^{1}$, M. Edington ${ }^{1}$, K. McFall ${ }^{2}$, E. Salina ${ }^{1}$, C. Diaper ${ }^{1}$, S. Drummond ${ }^{1}$, D. Tejwani ${ }^{1}$, M. E. Gregory ${ }^{1}$, J. Connolly ${ }^{1}$, P. Cauchi D $^{1}$, \\ K. Crofts ${ }^{1}$ and V. Chadha ${ }^{1 凶}$
}

(c) The Author(s), under exclusive licence to The Royal College of Ophthalmologists 2021

BACKGROUND/AIMS: Oculoplastics is a predominantly visual specialty and many of the pathologies can be diagnosed based on external appearance. An image-based eyelid lesion management service was piloted to reduce the number of patients who would require outpatient clinic review. The aim of this study was to determine its accuracy and feasibility, both as a hospital-based and community optometrist-based service. If successful, the service was envisaged to significantly reduce the number of patients that require face-to-face (F2F) review, in accordance with current post-COVID-19 principles of social distancing.

METHODS: Patients with lid lesions attending an oculoplastics clinic were assessed by consultant oculoplastic surgeons in an F2F consultation (Arm A). The lesions were photographed by a professional clinical photographer (Arm B) and by an optometrist with a handheld digital camera (Arm C). These images were reviewed by independent consultants masked to the outcome of the F2F clinical encounter. Data were collected prospectively including patient demographics, diagnosis, suspicion of malignancy and management. The image-based clinic results were compared to the F2F clinic results.

RESULTS: Ninety-five patients were included. Clinical diagnoses were compared for intra-observer variability and substantial agreement was demonstrated between gold-standard F2F clinic visit (Arm A) and Arm B $(K=0.708)$ and C $(K=0.776)$. There was no statistically significant difference in the rate of discharge and all cases of malignancy were either identified or flagged for F2F review in the image-based arms.

CONCLUSION: This pilot demonstrated substantial diagnostic agreement of image-based diagnoses with F2F consultation and image review alone did not miss any cases of malignancy.

Eye (2022) 36:1314-1318; https://doi.org/10.1038/s41433-021-01632-9

\section{INTRODUCTION}

The COVID-19 pandemic has highlighted the need for alternative ways to provide safe and efficient healthcare while respecting social distancing guidelines. In many specialties, this has required significant reductions in clinic numbers, more-efficient use of clinical space and appointment time, and increased reliance on telephone and video consultations.

External eye disease is a predominantly visual specialty and many conditions can be accurately diagnosed based on external appearance. Previous studies have examined image-based consultations in different contexts, including post-operative lid surgery, red-eye assessment, or eyelid tumour identification by medical students [1-3]. These have not assessed the validity of this modality for diagnosis and management of newly referred eyelid lesions.

The oculoplastic department in NHS Greater Glasgow \& Clyde (NHSGGC) sought to develop a new image-based eyelid lesion management service which, if successful, would likely significantly reduce the number of patients that need to be seen in the eye clinic. The aim of this study was to assess the accuracy and feasibility of a pilot of this new service, by determining whether diagnosis and management plan formulated from a photograph could be validated against the gold standard of a face-to-face (F2F) clinic consultation.

A secondary aim was to assess whether accurate and appropriate images would need to be taken by professionally trained clinical photographers in the hospital or could be provided by community-based optometrists taking photographs with a compact digital camera, thereby reducing the need of the patient to visit the hospital.

\section{METHODS}

Patients with lid lesions who were referred to the minor lid lesion clinic were seen by an oculoplastic consultant in accordance with normal practice. This was designated 'Arm A' and was deemed the 'gold standard' for comparison. At this clinic encounter, each patient had two clinical photographs taken -one by a clinical photographer (Arm B) and one by an optometrist who had undergone basic photography training (Arm C). This is illustrated in Fig. 1.

The clinical photographs were reviewed at a later date by separate, independent oculoplastic consultants who made a clinical diagnosis and management plan based on the referral, history and images. These consultants were masked to the clinical examination findings and outcome of the F2F clinical encounter. Prospective data collected included age, sex,

\footnotetext{
${ }^{1}$ Tennent Institute of Ophthalmology, Gartnavel General Hospital, Glasgow, UK. ${ }^{2}$ Head of Medical Illustration Services, NHS Greater Glasgow and Clyde, Glasgow, UK. 凶email: vchdh@aol.com
}

Received: 2 December 2020 Revised: 11 May 2021 Accepted: 10 June 2021

Published online: 25 June 2021 
All patients on the waiting list for Minor Ops/ Eyelids are sent a letter inviting

them to contact us for an appointment

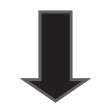

Outpatient clinic visit

\begin{tabular}{|c|c|c|}
\hline Arm A & Arm B & Arm C \\
\hline $\begin{array}{c}\text { Reviewed in face-to-face } \\
\text { Oculoplastic by Consultant }\end{array}$ & $\begin{array}{c}\text { Reviewed by Clinical } \\
\text { Photographer: Brief } \\
\text { history and clinical } \\
\text { photograph taken. }\end{array}$ & $\begin{array}{c}\text { Reviewed by Hospital } \\
\text { Optometrist: Brief history } \\
\text { and clinical photograph } \\
\text { taken. }\end{array}$ \\
\hline
\end{tabular}

\begin{tabular}{|c|c|c|}
\hline $\begin{array}{c}\text { Clinical diagnosis and } \\
\text { management plan } \\
\text { formulated. }\end{array}$ & $\begin{array}{c}\text { Clinical information and } \\
\text { photograph reviewed by } \\
\text { independent consultant } \\
\text { ophthalmologist, } \\
\text { If indicated, minor } \\
\text { procedure performed. }\end{array}$ & $\begin{array}{c}\text { Clinical information and } \\
\text { photograph reviewed by } \\
\text { second independent } \\
\text { formulated. }\end{array}$ \\
& $\begin{array}{c}\text { consultant } \\
\text { ophthalmologist, } \\
\text { management plan } \\
\text { formulated. }\end{array}$ \\
\hline
\end{tabular}

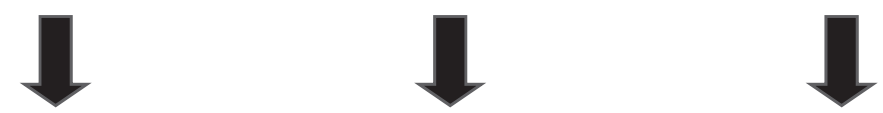

\begin{tabular}{|l|c|c|c|}
\hline $\begin{array}{l}\text { Clinical } \\
\text { outcome (N=95) }\end{array}$ & Arm A & Arm B & Arm C \\
\hline Discharge & $38(40 \%)$ & $\mathrm{N}(\%)$ & $27(28.4 \%)$ \\
\hline Minor ops & $47(49.5 \%)$ & $49(51.6 \%)$ & $43(45 \%)$ \\
\hline Main theatre & $8(8.4 \%)$ & $30(32 \%)$ & 0 \\
\hline Monitor & $2(2.1 \%)$ & 0 & $11(12 \%)$ \\
\hline $\begin{array}{l}\text { Outpatient } \\
\text { clinic }\end{array}$ & $\mathrm{NA}$ & $18(18.9 \%)$ & $14.7 \%)$ \\
\hline
\end{tabular}

Fig. 1 Outline of methodology and outcomes. Clinical pathway and outcomes for patients attending eyelid lesion management service.

provisional diagnosis and management (from F2F consultation; and based on each photography arm) and, where available, pathological diagnosis.

Diagnoses were classified into categories (see Table 1). The diagnosis from the 'gold standard' F2F clinic visit was compared with the diagnosis from Arms $B$ and $C$ by the calculation of the kappa coefficient to identify any intraobserver variability [4]. The scale used was as follows: Kappa < 0: no agreement; 0.0-0.21: slight agreement; $0.21-0.40$ : fair agreement; $0.41-0.60$ : moderate agreement; 0.61-0.80: substantial agreement; 0.81-1.0: almost perfect agreement. This was compared with the diagnosis and management of the patient in the clinic to determine the accuracy of the image-based system.

This study required no ethical approval as the clinical assessment and photography are conducted as part of the routine management of these patients. Patients consented to both sets of photos being additionally assessed by different clinicians. The NHS Health Research Authority online decision tool confirmed that no ethical approval was required as this study was conducted during routine clinical care. This study adhered to the tenets of the Declaration of Helsinki. Approval from the Caldicott guardian was obtained.

\section{RESULTS}

Ninety-seven patients with lid lesions were seen in these clinics, 49 $(50.5 \%)$ of which were male. The mean age was 53 years (range 
17-91). Two patients were excluded from further analysis as only one set of photos was taken.

The clinical diagnosis for each patient at clinic review was compared with the clinical diagnosis based on photography and history for Arm B and C with separate consultants (see Table 1). There was substantial agreement between diagnosis reached by clinicians in Arm A (clinic) and Arm B $(K=0.72 ; 95 \% \mathrm{Cl}$ 0.614-0.825) and also between Arm A (clinic) and Arm C ( $K=0.787,95 \% \mathrm{Cl} 0.698-0.884)$.

There were 10 lesions identified on $\mathrm{F} 2 \mathrm{~F}$ clinic review as suspected malignancy, of whom pathology confirmed a basal cell carcinoma in 8 (80\%). The other two lesions were identified as benign on pathology. All of these 10 lesions were also identified as suspicious by the clinicians reviewing the images from both Arm B (clinical photographer photos) and Arm C (optometrist photos). An example of images demonstrating benign and malignant pathology from each arm of the study is shown in Fig. 2. Agreement in determining malignancy was calculated using kappa coefficients, with the substantial agreement between Arm B and gold-standard F2F consultation $(K=0.7,95 \% \mathrm{Cl} 0.498-0.904)$ and almost perfect agreement between Arm $\mathrm{C}$ and gold-standard F2F consultation $(K=1.0)$

Table 1. Clinical diagnosis from arm A (F2F); arm B (clinical photographer) and arm C (optometrist).

\begin{tabular}{|c|c|c|c|}
\hline & $A(F 2 F)$ & B & C \\
\hline $\begin{array}{l}\text { Cystic lesions: cyst of Moll/Zeiss/Milia/ } \\
\text { epidermal/sebaceous cyst }\end{array}$ & 26 & 24 & 28 \\
\hline Chalazion/lesion related to chalazion & 35 & 29 & 34 \\
\hline Papilloma/skin tag & 18 & 29 & 15 \\
\hline Suspected malignancy & 10 & 17 & 10 \\
\hline $\begin{array}{l}\text { Other: naevus/vascular lesion/actinic keratosis/ } \\
\text { seborrhoeic keratosis }\end{array}$ & 6 & 3 & 8 \\
\hline Unsure, needs clinic review & NA & 2 & 2 \\
\hline
\end{tabular}

The outcome of the clinical encounter from clinic is documented in Fig. 1, and has been compared to both arms of the study. Forty percent of the patients were discharged without surgical intervention from the clinic. However, in Arm B, immediate discharge was recommended in $51.6 \%$, whereas in Arm $C$ it was recommended in $28.4 \%$. These differences were not statistically significant (Fisher's exact test Arm B vs $A p=0.145$ and Arm C vs A 0.09).

\section{DISCUSSION}

Resource management in the NHS involves identifying those patients who need care most, and allocating said resources appropriately. Resources have been stretched over recent years with increasing new patient referrals translating into increased waiting lists for clinic appointments [5]. This has been further impacted by the suspension of elective services during the first wave of the SARS-COV2 pandemic and reduced clinical capacity following on from this due to social distancing requirements in clinical areas, as well as the wish to avoid unnecessary hospital appointments which would increase exposure risk to our patients.

The oculoplastic 'minor operations' clinic has been identified as suitable for development into a virtual, image-based service. Some of these patients are referred unnecessarily with self-limiting conditions (such as recent onset chalazia) and some are conditions, which are purely cosmetic therefore are not eligible for excision under the NHS (such as small papillomas). However, occasionally patients referred with a 'benign' lesion may turn out to be malignant and, therefore, the key to adapting this service is to ensure these are always identified and treated appropriately.

Smartphone-based image quality was assessed by Boissin et al. [6] and deemed to be of comparable quality to digital cameras in a masked trial to rate images as 'good' or 'excellent' quality, concluding that they were an appropriate substitute for medical teleconsultation. The diagnostic accuracy of images has been closely linked to image quality in previous studies of burns and dermatological conditions $[7,8]$. We did not evaluate the quality of images but concentrated on the clinical relevance and found equivalent results amongst all arms of the study.
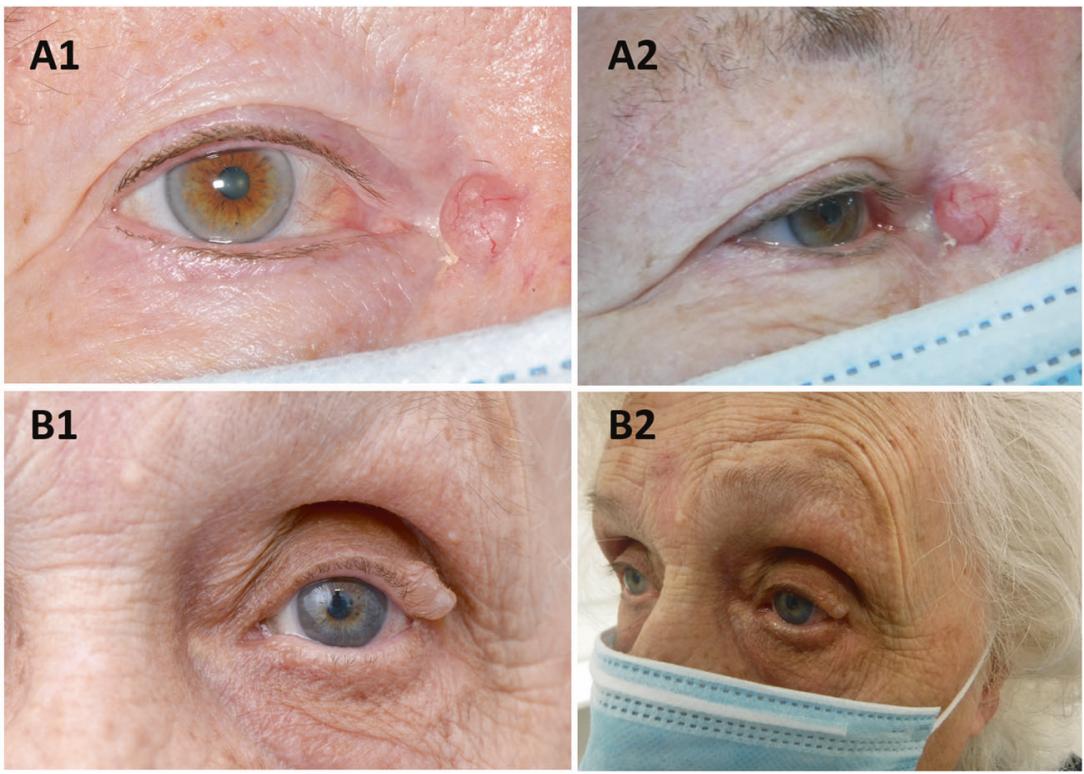

Fig. 2 Images taken by clinical photographers and optometrists. A True positive-basal cell carcinoma; images from clinical photographer (A1) and optometrist (A2). A diagnosis of BCC was made by clinicians reviewing images in both arms. This was in agreement with the F2F consultation and was subsequently confirmed by pathology. B False-positive-cyst of Moll; images from clinical photographer (B1) and optometrist (B2). The clinicians reviewing images in both arms felt that they were unable to exclude a BCC and therefore F2F review was recommended. A diagnosis of Cyst of Moll was made at F2F consultation and the patient discharged. 
The accurate identification of malignant lesions is essential to the safety and therefore success of an imaged-based 'virtual clinic' in oculoplastics. Leung et al. [9] developed a screening tool to allow the identification of malignant lesions by medical students from examination of a photograph. Seventy percents of malignant lesions were correctly identified. Our study involved expert consultant oculoplastic surgeons from whom we would assume and expect greater diagnostic clinical acumen and skill. This was borne by the results in which $100 \%$ of the malignancies were identified from the examination of photographs.

Within ophthalmology, previous studies have compared the diagnostic accuracy of smartphone-based images with traditional ocular exam findings and conventional images [1, 2]. Sink et al. [2] compared smartphone-based images taken by third party with ocular exam findings for diagnostic agreement in red eye and post-operative oculoplastic (lid) surgery complications, finding broad (red eye 15/16) or complete (post-op 16/16) agreement in diagnosis. Aoki et al. [1] compared the accuracy of lid tumour diagnosis via smartphone and camera images. Inperson evaluation was not statistically more accurate than teleevaluation and there was intra-observer agreement with the diagnosis of malignancy in this cohort of 36 patients. These studies are small and do not discuss the safety of reviewing new patient referrals with 'lid lumps' in a virtual image-based clinic.

In our study, we compared the clinical accuracy of diagnosing eyelid lesions in an F2F consultation with consultant oculoplastic surgeons (Arm A) with that of diagnoses made on a virtual review of referral, history and images by consultant oculoplastic surgeons. These images were taken by a professional clinical photographer using specialist camera equipment (Arm B) and by optometrists using a compact digital camera (Arm C), the latter representing the likely situation in community optometry. There was a substantial agreement regarding the clinical diagnoses with the gold-standard F2F clinical diagnosis in both Arm B $(K=0.708)$ and $C(K=0.776)$. Of the eight patients with confirmed malignancy (basal cell carcinoma in all cases) in this cohort of 95 patients, all were identified by the imaging Arms B and $C$, with a high level of intra-observer agreement. Our results suggest that images taken by a clinical photographer in a hospital environment or by an optometrist with a compact digital camera can be used as a surrogate to an F2F appointment and do not compromise on safety, i.e., are unlikely to miss malignant lesions.

There was a variable rate of discharge from this clinic, although this did not reach statistical significance. Nevertheless, the higher rate of discharge from the arm where the photographs were taken by clinical photographers makes it a more attractive service development in the NHS as it suggests the possibility of a halving the number of clinic appointments. One would expect this rate of discharge to be mirrored in the F2F clinic; however, doctor and patient are more likely to proceed with surgical intervention rather than conservative management in the physical environment of a one-stop clinic where the procedure can be done on the same visit.

The total number of hospital visits is not reduced by the development of medical illustration-based services. However, it results in a significant increase in capacity in the F2F oculoplastic clinic allowing those patients who will benefit most to be seen. In the future, there is a possibility of using images taken by community optometrists to make a provisional diagnosis and triage the patient, thereby avoiding a hospital visit completely.

Our study is limited by the small number of malignant lesions in our cohort. A further drawback is that multiple different consultants participated in the arms of the study (although each arm was separately assessed). There may be different practice patterns between the clinicians, and this would be reflected in the variable discharge rate. Finally, one could argue that we did not biopsy all the lesions and, in theory, a malignant lesion may have been missed in all arms. However, our study reflects an NHS practice in which benign lesions are often clinically diagnosed and all are not necessarily biopsied. We considered a diagnosis by an oculoplastics consultant on an F2F appointment as the gold standard and did not attempt to analyse the accuracy of that diagnosis.

In summary, our results validate the accuracy and safety of an image-based lid lesion management service. This is an attractive oculoplastics service development as it increases capacity, reduces unnecessary visits to the clinic, and improves the utilisation of consultant-led clinic appointments.

\section{Summary table}

What was known before:

- Image-based clinics have been studied in other specialties and deemed appropriate for remote consultation.

- Smartphone-based images have been demonstrated as a valid method of assessing post-operative oculoplastic surgery outcomes.

What this study adds:

- An image-based eyelid lesion management service is an accurate and safe method of virtually assessing lid lesions.

- These images can be taken by clinical photographers or provided by community optometry on referral, reducing the need for unnecessary clinic visits and maximising the utilisation of consultant-led oculoplastic clinics.

\section{REFERENCES}

1. Aoki L, Pereira IC, Matayoshi S. Comparative study between conventional camera images and smartphone images for eyelid tumor telediagnosis. Rev Col Bras Cir. 2019;46:e2083.

2. Sink J, Blatt S, Yoo D, Henry M, Yang SD, Vasaiwala R, et al. A novel telemedicine technique for evaluation of ocular exam findings via smartphone images. J Telemed Telecare. 2020;6:1357633X20926819.

3. Kumar S, Yogesan K, Constable IJ. Telemedical diagnosis of anterior segment eye diseases: validation of digital slit-lamp still images. Eye (Lond). 2009;23:652-60.

4. Landis JR, Koch GG. The measurement of observer agreement for categorical data. Biometrics 1977;33:159-74.

5. Elective Care Transformation Programme NHS England. Transforming elective care services: Ophthalmology. London. 2019. Accessed October 2020. https://www. england.nhs.uk/publication/transforming-elective-care-services-ophthalmology/

6. Boissin C, Fleming J, Wallis L, Hasselberg M, Laflamme L. Can we trust the use of smartphone cameras in clinical practice? laypeople assessment of their image quality. Telemed J E Health. 2015;21:887-92.

7. Roa L, Gómez-Cía T, Acha B, Serrano C. Digital imaging in remote diagnosis of burns. Burns 1999;25:617-23.

8. van der Heijden JP, Thijssing L, Witkamp L, Spuls PI, de Keizer NF. Accuracy and reliability of teledermatoscopy with images taken by general practitioners during everyday practice. J Telemed Telecare. 2013;19:320-5.

9. Leung C, Johnson D, Pang R, Kratky V. Identifying predictive morphologic features of malignancy in eyelid lesions. Can Fam Physician. 2015;61:e43-9.

\section{ACKNOWLEDGEMENTS}

The authors would like to acknowledge the support and assistance of the following colleagues in the development of this pilot clinic and acquisition of images: Jim Bretherton, Donna McCarter, Margaret Sharkey, Lynne Campbell, Tony Johnston, Kirsty Lattka, Andrew McCallister, James Eyland, John McCormack, Frances Barrett, and Shivani Tandon. 
AUTHOR CONTRIBUTIONS

V.C. conceived and designed the study. Data were acquired and analysed by K.M., E.S., C. D., S.D., D.T., M.E.G., J.C., P.C., K.C. and V.C. J.H. analysed and interpreted the data. J.H., V.C. and M.E. prepared the draft manuscript, which was revised and approved by all authors.

\section{FUNDING}

There are no funding sources to declare.

\section{COMPETING INTERESTS}

The authors declare no competing interests.

\section{CONSENT}

Consent for the publication of images has been obtained.

\section{ADDITIONAL INFORMATION}

Correspondence and requests for materials should be addressed to V.C.

Reprints and permission information is available at http://www.nature.com/ reprints

Publisher's note Springer Nature remains neutral with regard to jurisdictional claims in published maps and institutional affiliations. 